emphasize the importance of an accurate joint examination in these patients. The OSd group showed more joint manifestations which might be explained by the coexistence of SLE and MCTD patients in this group. Currently, no association between the clinical subtypes of IIM, overall, these results are encouraging and suggest that joint assessment in follow up may be helpful in differentiating subtypes of IIM.

References:

[1] Bohan A, Peter JB. N Engl J Med 1975 Feb 13;292(7):344-7.

Disclosure of Interest: None declared

DOI: 10.1136/annrheumdis-2017-eular.7051

\section{FRI0386 THE VALIDATION OF THE SCLERODERMA HEALTH ASSESSMENT QUESTIONNAIRE IN TURKISH SPEAKING PATIENTS WITH SYSTEMIC SCLEROSIS}

D. Temiz Karadag ${ }^{1}$, F. Karakas ${ }^{2}$, S. Tekeoglu ${ }^{1}$, O. Ozdemir Işik ${ }^{1}$, A. Yazici ${ }^{1}$, A. Cefle ${ }^{1} .{ }^{1}$ Rheumatology; ${ }^{2}$ Internal Medicine, Kocaeli University Medicine School, Kocaeli, Turkey

Background: The Health Assessment Questionnaire (HAQ) is one of the main instruments for assessing disability in rheumatic diseases.The Scleroderma $\mathrm{HAQ}$ (S-HAQ) combines the HAQ with five systemic sclerosis (SSc) related visual analogue scales for Raynaud's phenomenon (RP), digital ulcers, digestive symptoms, pulmonary symptoms, and overall disease severity.

Objectives: To perform cross-cultural adaptation and validation of the S-HAQ in patients with SSc.

Methods: Sixty patients who fulfilled the 2013 ACR/EULAR Classification Criteria for Systemic Sclerosis ACR criteria for SSc were were recruited.Fifty three percent of the patients fulfilled the criteria for limited systemic sclerosis (IcSSc).We evaluated test-retest reliability using the intraclass correlation coefficient (ICC); known-groups construct validity by stratifying patients according to severe organ involvement; and convergent validity using Spearman's correlation with mental and physical components of Short Form 36 version 2 (SF36v2).

Results: Eighty percent of the patients were female and the median age was 56 years old.Finger tip ulcers were observed in 16 patients $(26.7 \%)$ and severe organ involvements were detected as intersititial lung disease in $32(53 \%)$ and pulmonary hypertension in $5(8 \%)$ of the patients. HAQ Disability Index (HAQ-DI), digestive VAS, pulmonary VAS, digital ulcer VAS, Raynaud's phenomenon VAS and overall disease severity VAS demonstrated high reliability (ICCs $=0.819$ 0.908). The HAQ-DI showed higher correlation coefficients with physical function and role physical components of physical-related scores $(r=-0.537$ and -0.453 , respectively); social function and role emotional components of mental-related dimensions ( $r=-0.470$ and -0.328 , respectively) in the SF-36. Two among five SSc related VAS (VAS digital ulcer and VAS overall disease severity) were highly correlated with HAQ-DI ( $r=0.527$ and 0.481 respectively). The instruments could not discriminate between severe organ involvements and both subsets of SSc.

Table 1. Correlation of HAQ-DI with physical and mental components of SF-36 and, SSc related VAS scores

\begin{tabular}{lccc}
\hline $\mathrm{N}=60$ & Mean \pm SD and/or $\%$ & Correlation Coefficient & $p$-value \\
\hline Physical function & $55.4 \pm 23.83$ & -0.537 & 0.000 \\
Role physical & $33.82 \pm 42.67$ & -0.453 & 0.001 \\
Body pain & $53.82 \pm 28.84$ & -0.290 & 0.041 \\
General health & $39.9 \pm 18.43$ & -0.295 & 0.037 \\
Vitality & $41.08 \pm 18.39$ & -0.286 & 0.044 \\
Social function & $62.25 \pm 24.49$ & -0.470 & 0.001 \\
Role emotional & $49.02 \pm 32.9$ & -0.328 & 0.02 \\
Mental health & $61.12 \pm 18.39$ & -0.295 & 0.04 \\
VAS digestive & $0.39 \pm 0.65$ & 0.304 & 0.028 \\
VAS pulmonary & $0.64 \pm 0.79$ & 0.380 & 0.005 \\
VAS RP & $0.67 \pm 0.74$ & 0.354 & 0.01 \\
VAS digital ulcer & $0.62 \pm 0.94$ & 0.527 & 0.000 \\
VAS overall severity & $1.14 \pm 0.97$ & 0.481 & 0.000 \\
\hline
\end{tabular}

Conclusions: The Turkish version of the S-HAQ meet the requirements of reproducibility and validity.More studies are needed to examine the capacity of these instruments to discriminate between severe involvements and disease subsets. This study forms the basis for future studies to evaluate the properties of $\mathrm{S}-\mathrm{HAQ}$ in Turkish speaking SSc patients more extensively.

\section{References:}

[1] R Hesselstrand et al, Psychometric properties of the Swedish version of the Scleroderma Health Assessment Questionnaire and the Cochin Hand Function Scale in patients with systemic sclerosis, Scandjrheumatol Vol. 42, Iss. 4,2013.

Disclosure of Interest: None declared

DOI: 10.1136/annrheumdis-2017-eular.3289

\section{FRI0387 ANTI-RNA POLYMERASE III SUBSET OF SCLERODERMA PATIENTS: A MONOCENTRIC STUDY}

E. Pigatto, M. Ferranti, M. Favaro, P. Polito, E. Zanatta, P. Galozzi, M. Tonello, L. Punzi, F. Cozzi. University of Padova, Padova, Italy

Background: Systemic sclerosis (SSc) is an autoimmune disease characterized by heterogeneous clinical features and variable disease course. Specific antinuclear antibodies (ANA) identify different clinical subsets and are very useful in defining the prognosis of the patients. Recently, the anti-RNA polymerase III antibodies (anti-RNAP) were defined as the third specific ANA of SSc, together with anti-centromere (ACA) and anti-topoisomerase I (anti-Scl70).

Objectives: The aim of this study was to analyze the clinical picture of a group of SSc patients with anti-RNAP in a monocentric cohort of patients and to compare it with other subsets of patients with different specific ANA. In particular, the visceral involvement and the concomitant malignancies were investigated.

Methods: Among the SSc patients referring to the Rheumatology Unit of Padova University, 49 cases with anti-RNAP specific ANA were considered. Patient's demographics data and major clinical manifestations were compared with those of 50 ACA-positive and 52 anti-Scl70-positive patients. The skin score and the presence of digital ulcers, arthritis, interstitial lung disease (ILD), cardiac involvement including pulmonary hypertension, gastrointestinal disease (GI) and scleroderma renal crisis (SRC) were assessed. The presence and type of concomitant malignancies were also evaluated.

Results: $53 \%$ of anti-RNAP-positive patients had a diffuse cutaneous form of SSc. The skin score was significantly higher than in ACA-positive patients $(p<0.001)$. Digital ulcers were active in $73.5 \%$ of cases, without significant difference with the other two subsets. Arthritis was found in $20.4 \%$ of cases in comparison to $4 \%$ in ACA-positive patients $(\mathrm{p}<0.02)$. Regarding visceral involvements, $\mathrm{Gl}$ disease was the most common manifestation in all subsets; SRC was observed in $24.4 \%$ of anti-RNAP-positive patients and was significantly more frequent in comparison to ACA-positive $(p<0.005)$; ILD showed a prevalence of $42.8 \%$ in anti-RNAP subset, lower than in anti-Scl70-positive $(p<0.05)$ and higher than in ACA-positive patients $(p<0.01)$; cardiac involvement was observed in $32.6 \%$ of anti-RNAP-positive patients, without significant difference with the other subsets. Malignancies were found in $33(67.3 \%)$ anti-RNAP-, 18 (36\%) anti-Scl70- and $7(14 \%)$ ACA- positive patients. The most common cancer-sites were breast $(36 \%)$, lung (14\%) and colon (10\%). The risk of developing cancer was higher in anti-RNAP-positive patients than in other subsets (OR:6.35).

Conclusions: Our data demonstrated that anti-RNAP specific antibodies can identify a subset of SSc patients characterized by a severe clinical picture, with a high prevalence of diffuse cutaneous form, SRC, cardiac involvement and ILD. These patients showed also an elevated risk of developing cancer. Our results were consistent with recent published papers on this topic $(1,2)$.

\section{References:}

[1] Terras S et al. RNA polymerase III autoantibodies may indicate renal and more severe skin involvement in systemic sclerosis. Int J Dermatol 2016; 55:882-5.

[2] Lazzaroni MG et al. Malignancies in patients with anti-RNA Polymerase III antibodies and Systemic Sclerosis: analysis of the EULAR Scleroderma Trials and Research Cohort and possible recommendations for screening. J Rheumatol 2017; pii: jrheum.160817.

Disclosure of Interest: None declared

DOI: 10.1136/annrheumdis-2017-eular.3292

\section{FRI0388 LONG-TERM TREATMENT WITH RITUXIMAB IN SYSTEMIC SCLEROSIS PATIENTS: UPDATE OF OUR CLINICAL EXPERIENCE}

F. Lumetti, M. Colaci, C. Esposito, E. Cocchiara, C. Vacchi, A. Spinella, G. Tiengo, D. Giuggioli, C. Ferri. Scleroderma Unit, Chair of Rheumatology, University of Modena and Reggio Emilia, Policlinico of Modena, Modena, Italy

Background: Systemic sclerosis (SSc) is an immune-mediated disorder characterized by abnormal fibrosis and diffuse microangiopathy with skin and internal organ involvement. The treatment of SSc represents a great clinical challenge because of the complex disease pathogenesis including vascular, fibrotic, and immune T- and B-lymphocyte-mediated alterations. Therefore, SSc should be treated by combined or sequential therapies according to prevalent clinico-pathogenetic phenotypes. Some preliminary data suggest that rituximab (RTX) may be usefully employed in SSc patients.

Objectives: The present study aimed to evaluate the efficacy of RTX in our SSc patients' series as well as the long-term effects of this treatment.

Methods: A series of $15 \mathrm{SSc}$ patients (M/F 6/9, mean age $52.7 \pm 17.9 S D$ years, mean disease duration $10.3 \pm 7.1 \mathrm{SD}$ years, $\mathrm{L} / \mathrm{D}$ cutaneous subsets $5 / 10$ ) were treated with one or more cycles of RTX (4 weekly infusions of $375 \mathrm{mg} / \mathrm{m}^{2}$ ). In all patients RTX was repeated every 6 months for a total of 2-6 cycles. Patients' clinical-serological evaluation, including the self-evaluation of quality of life by means of $\mathrm{HAQ}$ and visual analogical scale (VAS) assessment, was performed every 6 months for a mean follow-up period of $42 \pm 24$ SD months.

Results: After the first 6 months following RTX treatment the extent of skin sclerosis measured with modified Rodnan skin score (mRSS) significantly improved (from $17.3 \pm 10.4$ to $13.4 \pm 7.6 ; p<.01$ ), and remained stable at the end of the follow-up $(13.3 \pm 8.1 ; p=.009)$. The usefulness of RTX on skin sclerosis was more evident in patients with diffuse cutaneous SSc $(n=10)$ showing a significant decrease of mRSS after the first 6 months (from $24.2 \pm 5.1$ to $18.1 \pm 4.7 ; p=.006$ ) and at the end of the follow-up period $(18.0 \pm 6.0 ; p=.005)$. Similarly, a valuable improvement of other cutaneous manifestations, namely hypermelanosis $(12 / 12$ pts), pruritus (11/13 pts), and calcinosis ( $3 / 6$ pts) was observed. Moreover, arthritis revealed particularly responsive to RTX treatment leading to a clear-cut reduction of swollen and tender joints in 12/13 patients; while lung fibrosis detected in 
12/15 remained stable during the entire follow-up. These positive clinical changes were mirrored by the subjective improvement of patients' well being in all cases (HAQ from $1.45 \pm 0.49$ to $0.98 \pm 0.38$, VAS from $64.5 \pm 18.5$ to $37.5 \pm 8.6$ ). Finally, no significant side effects were observed.

Conclusions: The present study reinforces the previous trials showing the usefulness of RTX in the management of SSc patients, along with its good safety profile. The specific therapeutical activity of RTX, able to down-regulate the B-cell over expression, might explain its beneficial effects on some SSc clinical manifestations; in particular the improvement of both skin sclerosis and articular involvement, along with the possible stabilization of lung fibrosis.

Disclosure of Interest: None declared

DOI: 10.1136/annrheumdis-2017-eular.2235

\section{FRI0389 FOOT INVOLVEMENT IN PATIENTS WITH SYSTEMIC SCLEROSIS: A SINGLE-CENTRE REPORT}

G. Cuomo, D. Ruta, E. Frongillo, L. Pirro, A. Del Mastro, C. Romano.

Dipartimento Assistenziale Integrato di Medicina Interna, UOC Medicina Interna - Università degli Studi della Campania - Luigi Vanvitelli, Napoli, Italy

Background: Foot involvement can be a source of morbidity and disability in patients with systemic sclerosis (SSc). Some studies have previously reported severity, echographic and radiographic manifestations of foot involvement in SSc $(1,2)$.

Objectives: The aim of our study was to assess the nature and prevalence of foot problems in patients with SSc reporting a single centre experience.

Methods: A podiatrist and a rheumatologist assessed 81 (76 female) consecutive patients attending our SSc outpatient clinic. The mean age was 50 years (range $21-70)$. Thirteen (16\%) had diffuse cutaneous SSc with a median disease duration from Raynaud's Phenomenon of 5 years (range $3-19) ; 68$ (84\%) had limited cutaneous SSc with a median disease duration of 11.5 years (range 1-41). The overall median disease duration was 11 years (range 1-41). Thirty (37\%) were anticentromere antibodies positive, thirty five (43\%) anti-scl70 antibodies positive. The two investigators evaluated the presence of the following features: colour changes, pain, previous ulceration, current ulceration, pre-ulceration (discoloration and thinning of the skin), toenail changes, hyperkeratosis, calcinosis, onychomycosis, dry skin, skin sclerosis, warts, scleredema, flatfoot. The presence of paresthesias, cramps, metatarsalgia were also investigated.

Results: The diagram reports the prevalence of foot manifestations in the $81 \mathrm{SSc}$ patients.

Most SSc patients suffer from symptoms related to their feet, particularly dry skin (70\%), hyperkeratosis (plantar 58\%, finger V 63\%), Raynaund's phenomenon $(59 \%)$, cramps $(55 \%)$. No statistically significant differences were found between diffuse and limited SSc groups.

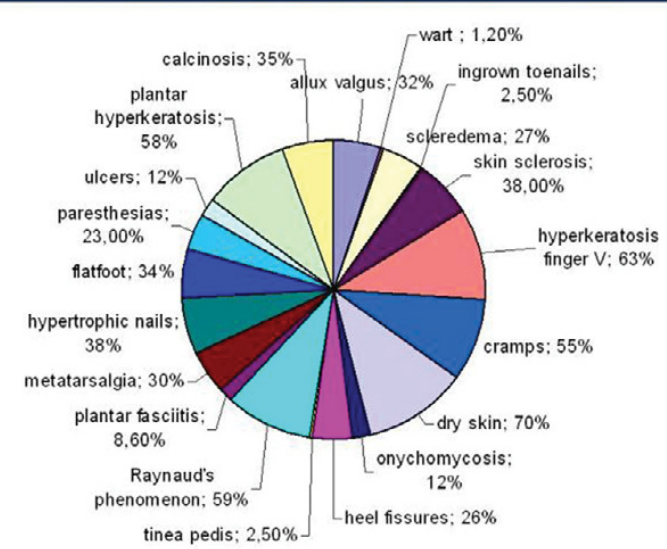

Prevalence of foot manifestations in the 81 SSc patients

Conclusions: Our study suggests that, in patients with SSc, foot problems are common and potentially disabling. A careful assessment of the feet should always be performed in these patients, in order to identify problems at an early stage. References:

[1] La Montagna G et al. Semin Arthritis Rheum 2002.

[2] Sari-Kouzel H et al. Rheumatology (Oxford) 2001.

Disclosure of Interest: None declared

DOI: 10.1136/annrheumdis-2017-eular.6097

\section{FRI0390 EUROPEAN MULTICENTRE STUDY VALIDATES ELF TEST AS BIOMARKER OF FIBROSIS IN SYSTEMIC SCLEROSIS}

G. Abignano $^{1,2}$, J. Blagojevic ${ }^{1,2,3}$, L.-A. Bissell ${ }^{1,2}$, R.B. Dumitru ${ }^{1,2}$, S. Eng ${ }^{1,2}$, Y. Allanore $^{4}$, J. Avouac ${ }^{4}$, S. Bosello ${ }^{5}$, C. Denton ${ }^{6}$, O. Distler ${ }^{7}$, G. Ferraccioli ${ }^{5}$ S. Jordan ${ }^{7}$, M. Matucci-Cerinic ${ }^{3}$, V. Ong ${ }^{6}$, M. Messenger ${ }^{8}$, M. Hutchinson $^{1}$,
M.H. Buch ${ }^{1,2}$, P. Emery 1,2, F. Del Galdo ${ }^{1,2} .{ }^{1}$ Leeds Institute of Rheumatic and Musculoskeletal Medicine, University of Leeds; ${ }^{2}$ NIHR Leeds Musculoskeletal Biomedical Research Unit, Leeds Teaching Hospitals NHS Trust, Leeds, United Kingdom; ${ }^{3}$ Department of Experimental and Clinical Medicine, Division of Rheumatology, University of Florence, Florence, Italy; ${ }^{4}$ Department of Rheumatology, University of Paris Descartes, Paris, France; ${ }^{5}$ Division of Rheumatology, Fondazione Policlinico Universitario Agostino Gemelli, Rome, Italy; ${ }^{6}$ Department of Rheumatology, University College London, Royal Free Hospital, London, United Kingdom; ${ }^{7}$ Department of Rheumatology, University Hospital Zurich, Zurich, Switzerland; ${ }^{8}$ NIHR Diagnostic Evidence Co-operative, Leeds Teaching Hospitals NHS Trust, Leeds, United Kingdom

Background: The Enhanced Liver Fibrosis (ELF) test is a serum test including the serum concentrations of amino-terminal pro-peptide of procollagen type III (PIIINP), tissue inhibitor of matrix metalloproteinase-1 (TIMP-1) and hyaluronic acid (HA). A recent single centre study showed that ELF score and its components are markers of overall fibrosis in systemic sclerosis (SSc) mainly reflecting skin and lung involvement (1).

Objectives: To determine the value of ELF score and its single analytes in an independent multicentre cohort of SSc patients.

Methods: 254 SSc patients from 6 European Rheumatology Centres were included in this study. Clinical data were collected at time of sampling. Serum samples were collected and stored according to EUSTAR biobanking recommendations (2). Sera were analysed employing a high-throughput in vitro diagnostic (Siemens Alpha-Centaur). Statistical analysis was performed with SPSS software V.24.

Results: The 254 SSc patients had a mean age $55.8 \pm 13.8$ years, and included 209 females and 80 patients with diffuse cutaneous SSc (dcSSc). ELF score was overall higher in males than in females $(\mathrm{p}=0.0236)$ as well as in dcSSc compared to limited cutaneous SSc patients $(p=0.0015)$. ELF score and the single markers significantly correlated with the degree of skin involvement (mRSS) and inversely correlated with FVC\%, TLC\% and DLCO\%. Concordantly all markers significantly correlated with skin and lung severity as assessed by the Medsger's scale (Table 1). TIMP-1 and PIIINP levels were higher in patients with lung fibrosis assessed by chest HRCT scan ( $p=0.0126$ and $p=0.0308$ respectively). Significant correlation $(p<0.0001)$ was found between ELF score, TIMP-1, PIIINP, HA and total disease severity and activity. Multivariate analysis indicated that age $(p<0.0001)$, mRSS $(p<0.0001)$ and DLCO\% $(p=0.005)$ were independently associated with ELF score.

Table 1. Coefficient correlation ( $r$ ) between ELF score, PIIINP, TIMP-1, HA serum levels and clinical variables

\begin{tabular}{llll}
\hline ELF score & PIIINP $(n g / m L)$ & TIMP-1 $(n g / m L)$ & $H A(n g / m L)$
\end{tabular}

Serum values

(median, range) $\quad 8.86,6.22-12.2 \quad 6.44,0.91-34.63 \quad 221,19.09-595.4 \quad 36.98,4.52-355.5$

\begin{tabular}{|c|c|c|c|c|}
\hline & $r$ & $r$ & $r$ & $r$ \\
\hline Age & $0.41^{\star \star \star \star \star}$ & 0.06 & $0.26^{\star \star \star \star}$ & $0.52^{\star \star \star \star}$ \\
\hline mRSS & $0.36^{\star \star \star \star}$ & $0.29^{\star \star \star \star}$ & $0.18^{\star \star}$ & $0.16^{*}$ \\
\hline FVC\% & -0.12 & $-0.25^{\star \star \star}$ & $-0.2^{\star \star}$ & 0.02 \\
\hline TLC\% & $-0.21^{\star \star}$ & $-0.29^{\star \star \star \star}$ & $-0.31^{\star \star \star \star}$ & -0.09 \\
\hline DLCO\% & $-0.25^{\star \star \star \star}$ & $-0.39^{\star \star \star \star}$ & $-0.30^{\star \star \star \star}$ & $-0.18^{\star \star}$ \\
\hline Sev skin & $0.28^{\star \star \star \star}$ & $0.27^{\star \star \star \star}$ & $0.22^{\star \star \star}$ & $0.17^{\star \star}$ \\
\hline Sev_lung & $0.25^{\star \star \star \star}$ & $0.29^{\star \star \star \star}$ & $0.3^{\star \star \star \star}$ & $0.18^{\text {** }}$ \\
\hline Sev_total & $0.32^{\star \star \star \star}$ & $0.27^{\star \star \star \star}$ & $0.33^{\star \star \star \star}$ & $0.23^{\star \star \star}$ \\
\hline EScSG-AI & $0.30^{\star \star \star \star}$ & $0.26^{\star \star \star \star}$ & $0.27^{\star \star \star \star}$ & $0.22^{\star \star \star}$ \\
\hline
\end{tabular}

Conclusions: The value of ELF score as independent marker of skin and lung involvement is confirmed in a second independent multicentre cohort of SSc patients. A longitudinal study paired with analysis of large cohort of healthy controls is currently on going to identify a SSc specific test with the highest predictive value for skin and lung progression independently of age and gender. References:

[1] Abignano G et al. Ann Rheum Dis. 2014.

[2] Beyer C et al. Ann Rheum Dis. 2011.

Disclosure of Interest: None declared

DOI: 10.1136/annrheumdis-2017-eular.3236

\section{FRI0391 A PERFUSION-METABOLIC MISMATCH IN 99MTL AND 123I-BMIPP SCINTIGRAPHY PREDICTS WORSE PROGNOSIS IN SYSTEMIC SCLEROSIS PATIENTS WITH ASYMPTOMATIC CARDIAC INVOLVEMENT}

H. lida, H. Hanaoka, T. Kiyokawa, Y. Takakuwa, T. Okazaki, H. Yamada, S. Ozaki Division of Rheumatology and Allergology, Department of Internal Medicine, St. Marianna University School of Medicine, Kawasaki, Japan

Background: Cardiac involvement is a manifestation of systemic sclerosis (SSc) that contributes to significant mortality and morbidity. Since many SSc patients with cardiac involvement are asymptomatic and it may progress silently, early diagnosis is still challenging. The perfusion-metabolic mismatch in cardiac scintigraphy indicates functional abnormality due to myocardial injury and has been expected to detect early cardiac involvement in various diseases. However, its clinical utility has been poorly evaluated in patients with SSc. 